\title{
INVERTIBLE MATRICES IN CERTAIN COMMUTATIVE SUBSEMIRINGS OF FULL MATRIX SEMIRINGS
}

\author{
N. Sirasuntorn ${ }^{1}$, R.I. Sararnrakskul ${ }^{2} \S$ \\ ${ }^{1,2}$ Department of Mathematics \\ Faculty of Science \\ Srinakharinwirot University
}

114 Sukhumvit 23, Wattana District, Bangkok 10110, THAILAND

\begin{abstract}
Then every field is a semifield. For a semifield $S$, we let $D_{n}(S)$ denote the set of all $A \in M_{n}(S)$ of the form

$$
\left[\begin{array}{ccccc}
x_{1} & 0 & \ldots & 0 & y_{1} \\
0 & x_{2} & \ldots & y_{2} & 0 \\
\ldots & \ldots & \ldots & \ldots & \ldots \\
0 & y_{2} & \ldots & x_{2} & 0 \\
y_{1} & 0 & \ldots & 0 & x_{1}
\end{array}\right]
$$

where $M_{n}(S)$ is the full $n \times n$ matrix semiring over $S$. Then $D_{n}(S)$ is a maximal commutative subsemiring of the semiring $M_{n}(S)$. If $S$ is a field, it is known that $A \in D_{n}(S)$ is invertible if and only if $\operatorname{det} A \neq 0$. In this paper, invertible matrices in $D_{n}(S)$ where $S$ is a semifield which is not a field are characterized. It is shown that if $S$ is a semifield which is not a field, then $A \in D_{n}(S)$ is an invertible matrix over $S$ if and only if $\left(x_{i}=0\right.$ if and only if $\left.y_{i} \neq 0\right)$.
\end{abstract}

AMS Subject Classification: 16Y60, 20M17, 15A09

Key Words: semiring, semifield, full matrix semiring, invertible matrix

\section{Introduction and Preliminaries}

A semiring $S$ is an algebraic structure $(S,+, \cdot)$ such that $(S,+)$ and $(S, \cdot)$ are semigroups and $\cdot$ is distributive over + . A semiring $(S,+, \cdot)$ is called additively

Received: Octobere 21, 2015

Published: February 3, 2016

$\S_{\text {Correspondence author }}$
(C) 2016 Academic Publications, Ltd.

url: www.acadpubl.eu 
[multiplicatively] commutative if $x+y=y+x[x \cdot y=y \cdot x]$ for all $x, y \in S$. We say that $(S,+, \cdot)$ is commutative if it is both addtitively commutative and multiplicatively commutative. An element 0 of $S$ is called a zero of the semiring $(S,+, \cdot)$ if $x+0=x=0+x$ and $x \cdot 0=0=0 \cdot x$ for all $x \in S$ and by an identity of $(S+, \cdot)$ we mean an element $1 \in S$ such that $x \cdot 1=1 \cdot x=x$ for all $x \in S$. Note that a zero and an identity of a semiring are unique.

If a semiring $(S,+, \cdot)$ has a zero 0 [an identity 1 , we say that an element $x \in S$ is additively [multiplicatively] invertible over $S$ if there exists an element $y \in S$ such that $x+y=y+x=0[x \cdot y=y \cdot x=1]$. Note that such a $y$ is unique and may be written as $-x\left[x^{-1}\right]$.

A commutative semiring $(S,+, \cdot)$ with zero 0 and identity 1 is called a semifield if $(S \backslash\{0\}, \cdot)$ is a group. Then every field is a semifield. It is clearly seen that the following fact holds in any semifield.

Proposition 1.1. If $S$ is a semifield, then for all $x, y \in S, x y=0$ implies that $x=0$ or $y=0$.

Example $1.2([2])$. Let $\mathbb{R}$ be the set of all real numbers, $\mathbb{Q}$ the set of rational numbers, $\mathbb{R}^{+}=\{x \in \mathbb{R} \mid x>0\}, \mathbb{R}_{0}^{+}=\mathbb{R}^{+} \cup\{0\}, \mathbb{Q}^{+}=\{x \in \mathbb{Q} \mid x>0\}$ and $\mathbb{Q}_{0}^{+}=\mathbb{Q}^{+} \cup\{0\}$. Then $\left(\mathbb{R}_{0}^{+},+, \cdot\right)$ and $\left(\mathbb{Q}_{0}^{+},+, \cdot\right)$ are semifields which are not fields.

We note that both $\left(\mathbb{R}_{0}^{+},+, \cdot\right)$ and $\left(\mathbb{Q}_{0}^{+},+, \cdot\right)$ are semifields which are not fields. These semifields have the property that 0 is the only additively invertible element, that is, for $x, y \in S, x+y=0$ implies $x=y=0$. In fact, this property is generally true.

Proposition $1.3([3])$. If $S$ is a semifield which is not a field, then 0 is the only additively invertible element of $S$.

A maximal commutative subsemiring of a semiring $S$ is defined naturally to be a maximal element of the set of all proper commutative subsemirings of $S$ under inclusion. If $S$ is a noncommutative ring, then a maximal commutative subsemiring of $S$ is a maximal element of the set of all commutative subsemiring of $S$ under inclusion.

For a positive integer $n$ and an additively commutative semiring $S$ with zero, let $M_{n}(S)$ be the set of all $n \times n$ matrices over $S$. Then under the usual addition and multiplication of matrices, $M_{n}(S)$ is also an additively commutative semiring with zero and the $n \times n$ zero matrix over $S$ is the zero of the matrix semiring $M_{n}(S)$. For $A \in M_{n}(S)$ and $i, j \in\{1,2, \ldots, n\}$, let $A_{i j}$ be the entry of $\mathrm{A}$ in the $i \underline{\underline{t h}}$ row and the $j \underline{\underline{t h}}$ column.

In 2010, Sararnrakskul, Lertvijitsilp, Wassanawichit and Pianskool [1] prove 
that the ring $D_{n}(R)$ of all $A \in M_{n}(R)$ of the form

$$
\left[\begin{array}{ccccc}
x_{1} & 0 & \cdots & 0 & y_{1} \\
0 & x_{2} & \cdots & y_{2} & 0 \\
\cdots & \cdots & \cdots & \cdots & \cdots \\
0 & y_{2} & \cdots & x_{2} & 0 \\
y_{1} & 0 & \cdots & 0 & x_{1}
\end{array}\right]
$$

is a maximal commutative subring of the ring $M_{n}(R)$ where $R$ is a commutative ring.

Let $S=(S,+, \cdot)$ be a commutative semiring with zero 0 and identity 1 . An $n \times n$ matrix $A$ over $S$ is called invertible over $S$ if there is an $n \times n$ matrix $B$ over $S$ such that $A B=B A=I_{n}$ where $I_{n}$ is the identity $n \times n$ matrix over $S$. Note that such a $B$ is unique.

The purpose of this paper is to show that when a square matrix in $D_{n}(S)$ is invertible over $S$ where $S$ is a semifield. Moreover, $D_{n}(S)$ is a maximal commutative subsemiring of the semiring $M_{n}(S)$.

\section{The Subsemiring $D_{n}(S)$ of $M_{n}(S)$}

From now on, let $S$ be a semifield. Let $n \in \mathbb{N} \backslash\{1\}$ and $\Lambda=\left\{1,2, \ldots,\left\lfloor\frac{n}{2}\right\rfloor\right\}$.

Note 2.1. For $A \in M_{n}(S), A \in D_{n}(S)$ if and only if (i) $A_{i i}=A_{n-i+1, n-i+1}$ and $A_{i, n-i+1}=A_{n-i+1, i}$ for all $i \in \Lambda$ and (ii) $A_{i j}=0$ for all $i, j \in\{1,2, \ldots, n\}$ with $j \neq i$ and $j \neq n-i+1$.

By the proof of Lemma 2.2 and Theorem 2.3 in [1], we have more generalized result for $D_{n}(S)$ where $S$ is a semifield as the following theorem.

Theorem 2.2. The set $D_{n}(S)$ is a maximal commutative subsemiring of the semiring $M_{n}(S)$.

It is well-known that a square matrix $A$ over a field $F$ is invertible if and only if $\operatorname{det} A \neq 0$. Therefore if $S$ is a field and $A \in D_{n}(S)$ then $A$ is invertible if and only if $\operatorname{det} A \neq 0$. For this reason if $S$ is a semifield which is not a field and $A \in D_{n}(S)$ when $A$ is invertible. So, we characterize invertible matrices in a commutative subsemiring of the semiring $M_{n}(S)$ where $S$ is a semifield which is not a field.

Theorem 2.3. Let $S$ be a semifield which is not a field. Then $A \in D_{n}(S)$ is invertible if and only if every row and every column of $A$ contains exactly one nonzero element, that is, for each $i \in \Lambda, A_{i i}=0$ if and only if $A_{i, n-i+1} \neq 0$. 
Proof. It is evident if $n=1$. Assume that $n>1$ and $A \in D_{n}(S)$ is invertible. Let $B \in D_{n}(S)$ such that $A B=B A=I_{n}$. Then we have the following equalities for $i \in \Lambda$,

$$
\begin{aligned}
1=(A B)_{i i} & =\sum_{k=1}^{n} A_{i k} B_{k i} \\
& =A_{i i} B_{i i}+A_{i, n-i+1} B_{n-i+1, i}
\end{aligned}
$$

and

$$
\begin{aligned}
0=(A B)_{i, n-i+1} & =\sum_{k=1}^{n} A_{i k} B_{k, n-i+1} \\
& =A_{i i} B_{i, n-i+1}+A_{i, n-i+1} B_{n-i+1, n-i+1}
\end{aligned}
$$

By (2) and Proposition 1.3 we obtain that

$$
A_{i i} B_{i, n-i+1}=0 \text { and } A_{i, n-i+1} B_{n-i+1, n-i+1}=0
$$

If $A_{i i}=0$, then by (1) $A_{i, n-i+1} \neq 0$.

Assume that $A_{i, n-i+1} \neq 0$ and $A_{i i} \neq 0$. By $(3), B_{i, n-i+1}=0$ and $B_{n-i+1, n-i+1}$ $=0$. Since $B_{i i}=B_{n-i+1, n-i+1}=0$ and $B_{n-i+1, i}=B_{i, n-i+1}=0$, by (1) we have $A_{i i} B_{i i}+A_{i, n-i+1} B_{n-i+1, i}=0$ a contradiction. So, we have if $A_{i, n-i+1} \neq 0$ then $A_{i i}=0$.

Conversely, assume that for each $i \in \Lambda, A_{i i}=0$ if and only if $A_{i, n-i+1} \neq 0$. Define $B \in D_{n}(S)$ by

$$
B_{i j}= \begin{cases}A_{i j}^{-1} & \text { if } A_{i j} \neq 0, \\ 0 & \text { if } A_{i j}=0 .\end{cases}
$$

We will show that $A B=I_{n}$. The following equalities for $i \in \Lambda$ :

$$
\begin{aligned}
(A B)_{i i} & =\sum_{k=1}^{n} A_{i k} B_{k i} \\
& =A_{i i} B_{i i}+A_{i, n-i+1} B_{n-i+1, i} \\
& =A_{i i} B_{i i}+A_{i, n-i+1} B_{i, n-i+1} \\
& = \begin{cases}A_{i, n-i+1} B_{i, n-i+1} & \text { if } A_{i i}=0 \\
A_{i i} B_{i i} & \text { if } A_{i i} \neq 0\end{cases} \\
& =1 .
\end{aligned}
$$


and

$$
\begin{aligned}
(A B)_{i, n-i+1} & =\sum_{k=1}^{n} A_{i k} B_{k, n-i+1} \\
& =A_{i i} B_{i, n-i+1}+A_{i, n-i+1} B_{n-i+1, n-i+1} \\
& = \begin{cases}A_{i, n-i+1} B_{n-i+1, n-i+1} & \text { if } A_{i i}=0 \\
A_{i i} B_{i, n-i+1} & \text { if } A_{i i} \neq 0\end{cases} \\
& = \begin{cases}0 & \text { if } A_{i i}=0\left(\because B_{n-i+1, n-i+1}=B_{i i}=A_{i i}=0\right) \\
0 & \text { if } A_{i i} \neq 0\left(\because B_{i, n-i+1}=A_{i, n-i+1}=0\right) .\end{cases}
\end{aligned}
$$

Also if $i, j \in\{1,2, \ldots, n\}$ are such that $j \neq i$ and $j \neq n-i+1$, then by Note 2.1 , we have

$$
\begin{aligned}
(A B)_{i j} & =\sum_{k=1}^{n} A_{i k} B_{k j} \\
& =A_{i i} B_{i j}+A_{i, n-i+1} B_{n-i+1, j} \\
& =A_{i i} 0+A_{i, n-i+1} 0=0 .
\end{aligned}
$$

This shows that $A B(=B A)=I_{n}$. Hence $A$ is invertible.

Example 2.4. Let $n>1$ and

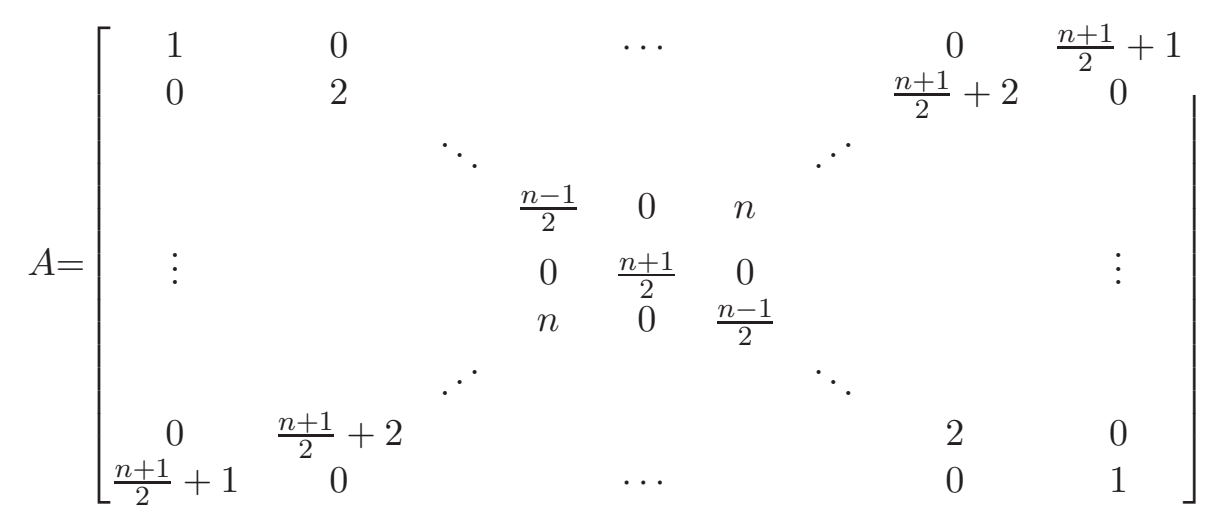

if $n$ is odd, and 


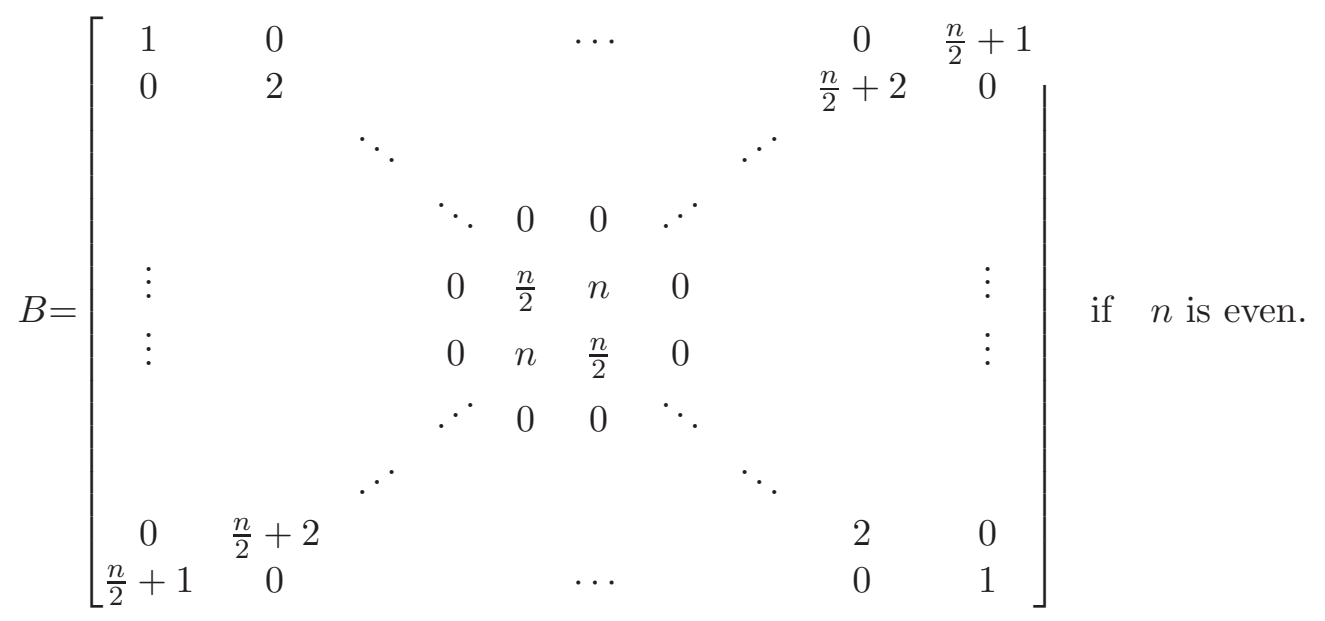

Clearly that $\operatorname{det} A \neq 0$ and $\operatorname{det} B \neq 0$, so $A$ and $B$ are invertible over a field $\mathbb{R}[\mathbb{Q}]$. However, by Theorem $2.3, A$ and $B$ are not invertible over a semifield $\left(\mathbb{R}_{0}^{+},+, \cdot\right)\left[\left(\mathbb{Q}_{0}^{+},+, \cdot\right)\right]$. If

$$
C=\left[\begin{array}{cccccccccc}
1 & 0 & 0 & & \cdots & \ldots & & 0 & 0 & 0 \\
0 & 0 & 0 & & \ldots & \ldots & & 0 & 2 & 0 \\
0 & 0 & 0 & & & & & 3 & 0 & 0 \\
& & & \ddots & & & . \cdot & & & \\
\vdots & \vdots & & & 0 & . \cdot & & & \vdots & \vdots \\
\vdots & \vdots & & & . \cdot & 0 & & & \vdots & \vdots \\
& & & . \cdot & & & \ddots & & & \\
0 & 0 & 3 & & & & & 0 & 0 & 0 \\
0 & 2 & 0 & & \ldots & \ldots & & 0 & 0 & 0 \\
0 & 0 & 0 & & \ldots & \ldots & & 0 & 0 & 1
\end{array}\right],
$$

then $C$ is invertible over the semifield $\left(\mathbb{R}_{0}^{+},+, \cdot\right)\left[\left(\mathbb{Q}_{0}^{+},+, \cdot\right)\right]$, so $C$ is invertible over the semifield $(\mathbb{R},+, \cdot)[(\mathbb{Q},+, \cdot)]$.

In 2014, N. Sirasuntorn and R. I. Sararnrakskul [4] show that the semiring $D V_{n}(S)$ of all $A \in M_{n}(S)$ of the form

$$
\left[\begin{array}{ccccc}
x_{1} & 0 & \cdots & 0 & x_{1} \\
0 & x_{2} & \cdots & x_{2} & 0 \\
\cdots & \cdots & \cdots & \cdots & \cdots \\
0 & x_{2} & \cdots & x_{2} & 0 \\
x_{1} & 0 & \cdots & 0 & x_{1}
\end{array}\right]
$$


is a regular commutative subsemiring of the semiring $M_{n}(S)$ is where $S$ is a regular semiring having some properties. Note that if $S$ is a semifield then $D V_{n}(S) \subsetneq D_{n}(S)$.

Theorem 2.5. Let $S$ be a semifield. Then the set $D V_{n}(S)$ is a commutative subsemiring of $D_{n}(S)$.

Proof. This proof is straightforward.

Corollary 2.6. Let $S$ be a semifield which is not a field. Then every element in $D V_{n}(S)$ is not invertible.

Proof. By Theorem 2.3 and the definition of the set $D V_{n}(S)$.

Acknowledgement The authors acknowledge the support of the SWU Endowment Fund Year 2014 through the reseach project, under contract number 112/2557, Srinakharinwirot University.

\section{References}

[1] R.I. Sararnrakskul, P. Lertwichitsilp, A. Wasanawichit, S. Pianskool, Certain maximal commutative subrings of full matrix rings, Chamchuri J. Math., 2, No. 1 (2010), 47-55.

[2] R.I. Sararnrakskul, S. Sombatboriboon, Invertible Matrices over semifields, East-West J. Math., 11, No. 2 (2009), 223-229.

[3] P. Sinutoke, Master Thesis: On the Theory of Semifields, Chulalongkorn University, Thailand (1980).

[4] N. Sirasuntorn, R.I. Sararnrakskul, Regularity of Certain Subsemirings of Full Matrix Semirings, East-West J. Math., 17, No. 1 (2015), 23-32. 
